$\Delta$ Palabras clave/ Cartografía histórica, Araucanía, paisaje, imaginario.

$\Delta$ Keywords/ Historical cartography. Araucanía, landscape, imaginary.

$\Delta$ Recepción/ 19 abril 2018

$\Delta$ Aceptación/ 13 agosto 2018

\title{
El paisaje araucano en la cartografía de 1575 a 1646: Una imagen del territorio basada en el dominio
}

\author{
Araucanian landscapes in cartography \\ between 1575-1646: \\ A domain-based image of the territory
}

RESUMEN/ La labor exploratoria de los navegantes, militares y misioneros de las compañías de conquista y colonia españolas que llegaron a la Araucanía se tradujo en una cartografía que trataba de localizar recursos, establecer defensas y controlar puntos clave del territorio. Este trabajo tiene por objetivo explicar el proceso de construcción de una imagen del territorio basada en el dominio, desarrollada desde la llegada de los españoles hasta la definición de la frontera del Biobío en 1646. La metodología se centra, fundamentalmente, en el estudio de mapas históricos, y consiste en el desarrollo de un análisis topográfico e iconográfico de la cartografía sumado a un posterior análisis crítico que da cuenta de las dificultades de carácter historiográfico que surgen, así como del hallazgo de mapas y cartas que han tenido escasa difusión o no han sido descritos con anterioridad. ABSTRACT/ The exploration endeavors by navigators, soldiers and missionaries who were part of the Spaniard colony and conquest companies that arrived in La Araucanía resulted in a cartography that attempted to identify resources, establish defenses and control key points in the area. This work is aimed at explaining the process of building a domain-based image of the territory, developed since the arrival of the Spaniards until the establishment of the Biobí border, in 1646. The methodology is mainly focused on studying historical maps and consists on the development of a topographic and iconographic analysis of cartography, in addition to a critical analysis about the historiographical issues that emerged, as well as the discovery of maps and charts that have been poorly disseminated or not described before.

\section{INTRODUCCIÓN Y ESTADO DEL}

\section{ARTE. Dentro de los estudios históricos} de cartografía, numerosos autores han señalado la relación crítica que existe entre la cartografía y la representación del territorio, en tanto esta constituye un ejercicio de poder donde se puede leer no solo aquello que se proclama en el documento, sino muchos otros elementos subyacentes que el propio plano revela (Kaplan 2013; Edney 2007; Harley, Laxton y Andrews 2005; Bousquet-Bressolier 1995; por citar algunos relevantes).
En el ámbito latinoamericano, algunos autores han centrado su trabajo en desvelar esta condición crítica (Sagredo 2013 Lois 2000; Kagan 2000; entre otros). En relación a la Araucanía, algunos autores han desarrollado estudios históricos de perspectiva amplia que comprendían la cartografía junto con el análisis de otras fuentes históricas (Guarda 1990, 1978). Otros se han centrado en el estudio del período republicano y la construcción de país (Antivil 2018; Flores y Azócar 2017; González Bernedo, 2013; Núñez 2012; Álvarez 2000).
En las últimas dos décadas, se ha iniciado una línea de estudio que trata de caracterizar los paisajes que han surgido históricamente y desvelar las dinámicas que han operado en el tiempo (Dillehay y Saavedra 2007; Alfaro 2014; Aliste 2011; Solari, Cueto, Hernández, Rojas y Camus 2011; Cisternas y Torrejón 2002). El presente trabajo se sitúa en esta misma línea. La investigación consiste en caracterizar y estudiar la evolución de la representación del paisaje en función de fuentes documentales cartográficas de la época, 
para contribuir a generar una lectura compleja de la Araucanía, donde los factores productivos, ideológicos e identitarios se sintetizan en una construcción cultural del territorio, es decir, en un paisaje (Maderuelo 2005). El propósito de este estudio consiste por lo tanto, en analizar la cartografía histórica de la Araucanía entre 1575 y 1646 bajo una mirada doble -cultural (Cosgrove y Daniels 1988) y morfológica (Cárcel 2016)- que permita analizar el territorio bajo la perspectiva paisajística. El dominio del territorio y el establecimiento de sus condiciones de producción constituyen un aspecto esencial del ejercicio del poder (Lefebvre 1974). La lectura crítica de la cartografía estudia el plano como correlato territorial de la circunstancia histórica, en tanto es un instrumento de poder y se utiliza como tal. La selección cartográfica se centra en ejemplos tempranos y paradigmáticos de representación del territorio, centrándose por primera vez en elementos del paisaje que, posteriormente, se han afianzado en la

\section{historia de la region.}

METODOLOGÍA. La metodología aplicada en la realización de este trabajo parte de las indicaciones de San-Antonio (2006) para realizar un análisis gráfico de cartografía histórica, quien se inspira, a su vez, en los estudios de Ernst H. Gombrich sobre la representación gráfica. Este método constituye una versión completa, sistemática y ordenada de los análisis que realizan actualmente tanto los archivos de referencia de este trabajo (Archivo General de Indias, Biblioteca Nacional de Chile, Biblioteca Nacional de España) como los principales estudiosos de la cartografía histórica nacional (González Leiva, Guarda, Martinic, González y Bernedo o Flores y Azócar, entre otros).
Las etapas de la metodología pueden ser ordenadas como sigue:

1. Confección de una ficha de catalogación que incluye: título, autor, fecha, escala, orientación, cartela, caligrafía, contenido, idioma, autor, ubicación, soporte, técnica y dimensiones.

2. Realización de un encuadre del plano: el marco geográfico, el contexto histórico, el estudio comparado con cartografía y textos escritos de la época y la figura del autor.

3. Descripción de su contenido, incluyendo las convenciones simbólicas, procedimientos de representación y perspectiva, elementos gráficos (cartelas, rosas de los vientos, escala, norte, orlas, etc.).

4. Realización de un ejercicio de síntesis en el que se establecen relaciones y mecanismos de causalidad entre el encuadre, el contenido representado y la representación gráfica, tanto desde el punto de vista de lo denotado como de las connotaciones semánticas interpretadas a partir de la puesta en relación de los factores analizados.

En este artículo se muestran tan solo las concusiones derivadas del estudio descrito.

DISCUSIÓN. La costa chilena fue la última en explorarse y cartografiarse de toda Sudamérica. Los primeros esbozos de la geografía de Chile que se conservan no comenzaron a realizarse hasta más de treinta años después de que los primeros exploradores mostraran el continente americano en las cortes europeas, a finales del siglo XV. Fue Fernando de Magallanes el primero en avistar Chile por el sur en 1520 y Diego de Almagro no accedió por el norte sino hasta 1534. La selección del material planimétrico se ha realizado partiendo de una perspectiva amplia, que trata de encuadrar el momento histórico en el que

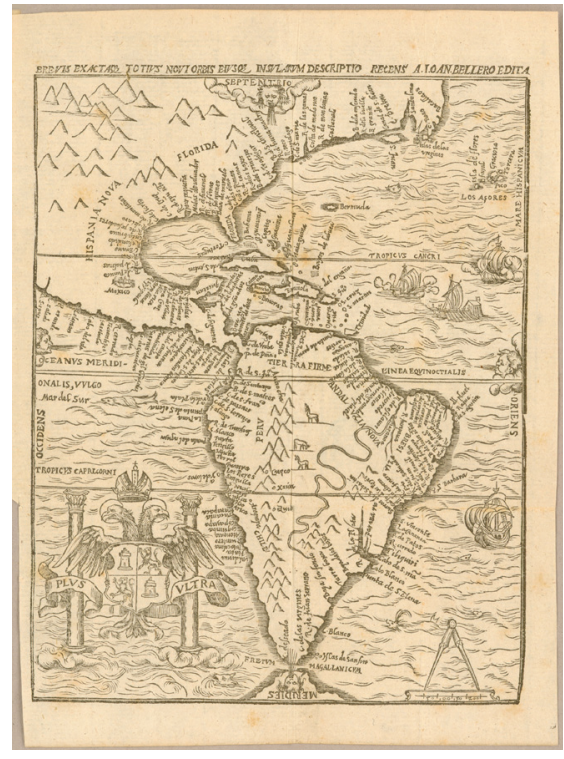

Imagen 1. Brevis exactaque totius Novi Orbis eiusque insularum descriptio recens a Joan. Bellliro [sic] edita, 1554 (fuente: John Carter Brown Library).

se comienza a trazar la geografía del Nuevo Mundo para centrarse, posteriormente, en la región. Recién a fines del siglo XVI se comenzó a representar Chile como unidad geográfica. Posteriormente, comenzaron las primeras tentativas de localización y descripción del territorio en torno a Concepción. A lo largo de este período, este puerto surgió y se consolidó rápidamente como lugar de refugio, comercio y aprovisionamiento para los navíos que superaban la peligrosa travesía del Cabo de Hornos y continuaban hacia la costa de Perú. En períodos posteriores, Concepción continuó siendo considerado como lugar de abrigo de buques que emprendían la exploración del Océano Pacífico. 
A partir de la llegada de Diego de Almagro en 1534, el territorio chileno terrestre comenzó a ser reconocido. Así, poco después de la fundación de Concepción en 1550 , se registraron por primera vez algunos topónimos de la costa. El mapa Brevis exactaque totius Novi Orbis eiusque insularum descriptio recens a Joan. Belllro [sic] edita de 1554 (imagen 1), ya incluye el término Chile y reconoce lugares como Valdivia (nombrada por Pastene en 1544 y fundada por Valdivia en 1552), Itata Quintero, Tacama (Atacama), Tarapaca (Tarapacá), Arica o Coquinba (Coquimbo), entre otros.

La primera vez que se representa el territorio chileno como una unidad territorial autónoma, es en el manuscrito titulado Descripción de la provincia de Chile de 1575 (imagen 2), de Juan López de Velasco. En él se dibuja la superficie comprendida entre el valle de Copiapó y el sur de Chiloé. Al norte limita con la Audiencia de los Charcas y al sur con las provincias del Estrecho. Chile se dibuja como una llanura apretada, larga y estrecha, entre la cordillera y el océano, fragmentada hacia el sur. Este territorio se ve surcado transversalmente por múltiples ríos, que se trazan con algunos afluentes. En este mapa aparece representada por primera vez la ciudad de La Concepción al borde de una bahía (de trazo impreciso y abierta al oeste) y los puertos de La Herradura (Dichato), Talguano (Talcahuano), Cañete, Cauten, además de otras poblaciones más al sur, como Los infa[n]tes, La Imperial, Villar[r]ica, Osorno, Valdivia y Castro. La toponimia comienza a precisarse y los principales ríos ya están representados: Maule, Itata (Ytata), Toltén (Tolten) y Valdivia. Los ríos Biobío e Imperial se dibujan -aunque no se nombran- y ambos se trazan con afluentes, especialmente el Biobío, donde se aprecia una bifurcación inicial que podría ser la confluencia con el Laja. López de Velasco se refiere al Biobio, en el libro Geografía y descripción universal de las Indias, de la siguiente manera: "Río grande de Biobio, en el cual entran, por la parte del norte el río grande de Niuequeten [Laja], y por la del sur el río de Vergara y de Angol, y el río de Tornacura, que todos tres se juntan antes de entrar en él, y los unos y los otros nacen de la cordillera" (López de Velasco y Fernández 1894). En el texto hay una descripción prolija de la geografía, los recursos naturales y los pobladores. La proximidad de las ciudades, acentuada en dirección sur, permite deducir el avance del sistema colonial, con una actividad fundacional progresiva y el intento de fijar población en los nuevos territorios. Se pretende asegurar el auxilio a los asentamientos de vanguardia mediante la proximidad de otras poblaciones (y fuertes, no señalados aquí) que puedan enviar tropas en caso necesario. Con

López de Velasco, la estructura esencial del territorio (cordillera paralela a la costa, grandes ríos perpendiculares) y el modelo de asentamiento de la conquista quedan vinculados por primera vez

La operación de conquista tuvo una gran resistencia y la cartografía del momento refleja la dificultad del dominio. El plano de 1605 incluido en el manuscrito titulado Relación del viaje de Fray Diego de Ocaña por el Nuevo Mundo (1599-1605) (imagen 3) forma parte del conjunto de planos que describen el territorio chileno, narrando el viaje de Ocaña por Sudamérica

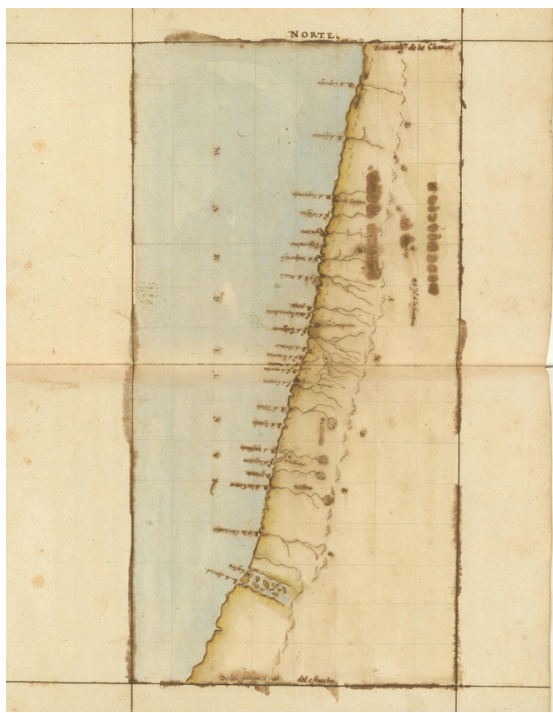

Imagen 2. Descripción de Provincia de Chile, 1575 (fuente: John Carter Brown Library)

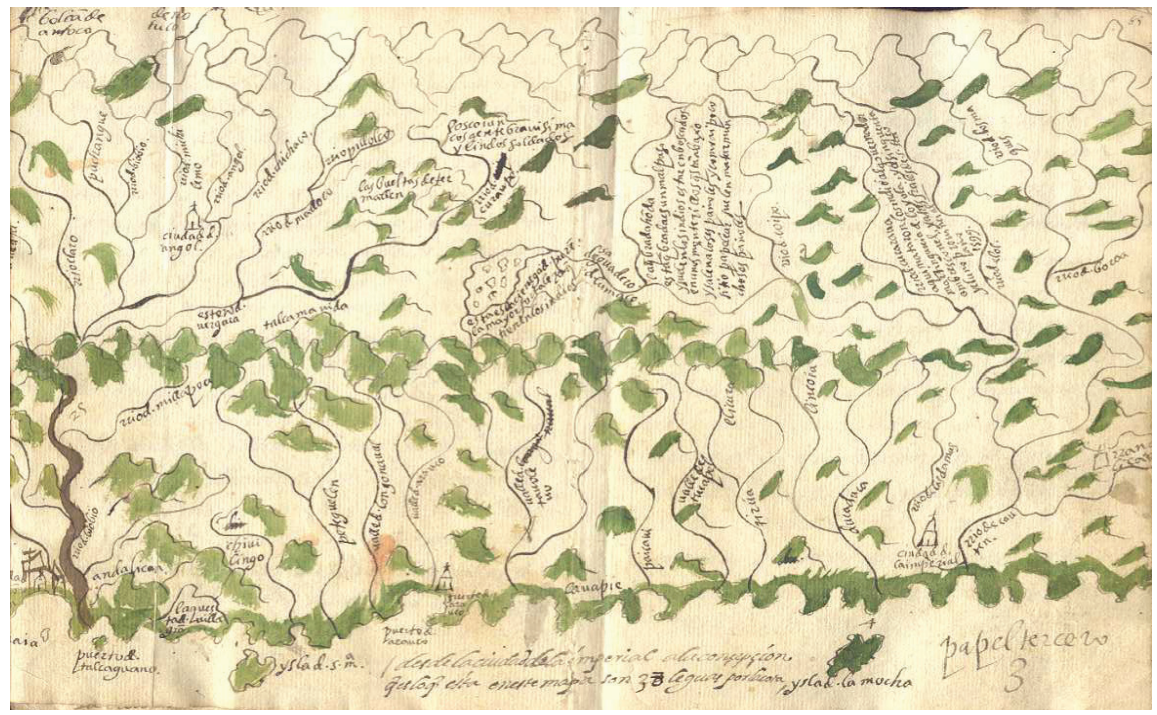

Imagen 3. Relación del viaje de Fray Diego de Ocaña por el Nuevo Mundo (1599-1605). Folio 85. (1605) (fuente: Biblioteca de la Universidad de Oviedo). 


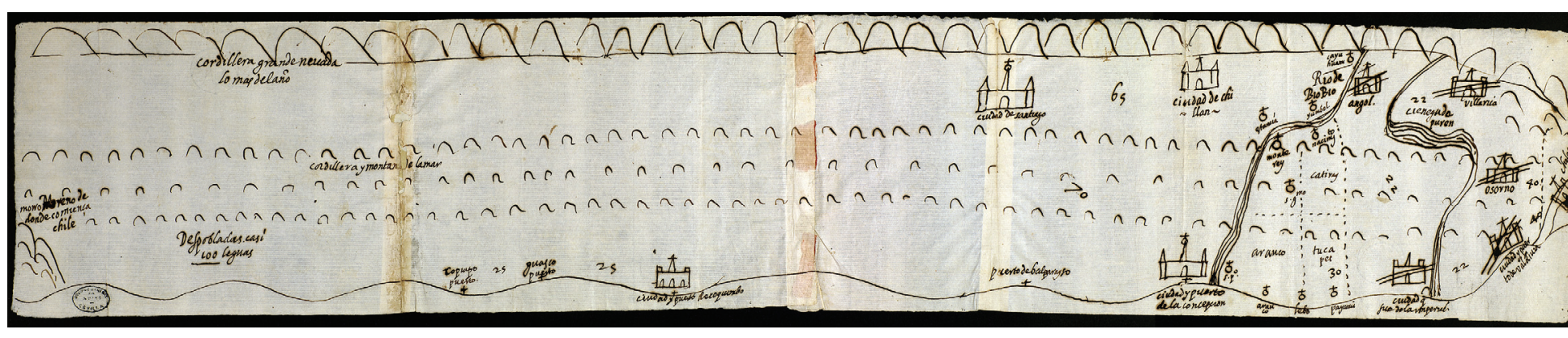

Imagen 4. Mapa del Reino de Chile, 1610 (fuente: Archivo General de Indias).

entre 1599 y 1605. Se trata de una de las primeras manifestaciones del conflicto entre españoles y mapuche. Este plano se descompone en cuatro fragmentos que abarcan desde Coquimbo hasta Chiloé, con franjas territoriales de entre 350 y $500 \mathrm{~km}$ de longitud. Se aprecia un conocimiento bastante preciso de la situación de los diferentes elementos del territorio y sus posiciones relativas. El interés documental reside en la representación del viaje de Ocaña en el año 1600, ilustrando la narración de los sucesos posteriores a la batalla de Curalaba y describiendo la pérdida de las ciudades de Valdivia, Angol, Chillán, Villarrica y Osorno a manos de los indígenas, mientras explica los peligros que sufrió el fraile durante su viaje. Si bien Peña (2016) pone en duda que Ocaña viajara realmente a la Araucanía, debido a ciertas contradicciones y errores en el texto, hemos interpretado que el texto tiene validez suficiente para explicar la percepción del territorio que se tenía en el momento histórico. La descripción es minuciosa, señalando accidentes geográficos, distancias e incluso las latitudes de algunos lugares. También informa de los modelos de asentamiento indígena, localiza recursos mineros y el aprovechamiento agrícola de ciertas zonas. Representa el carácter montuoso del territorio al sur del Biobío a lo largo de la Cordillera de la Costa (representada por primera vez) y el territorio de Arauco, señalando topónimos que aluden a la red fluvial y otros accidentes geográficos. También añade descripciones que previenen de emboscadas, marcando el lugar de sucesos históricos (matanza de jesuitas). Las plazas principales se representan a pesar de constatar en el texto la destrucción de algunas de ellas. De esta forma, el plano es crónica e ilustración de manera simultánea. El espacio físico se carga de significados asociados a la forma del territorio, combinado con los hechos experimentados. Se podría decir que, con Ocaña, la cartografía comienza a construir culturalmente el territorio, dando lugar a un paisaje con sentido histórico.

Otro mapa extraordinariamente relevante es el manuscrito Mapa del Reino de Chile, (imagen 4). Tal y como señala Alfaro (2014), forma parte del expediente presentado al Consejo de Indias y la Junta de Guerra en 1610 para argumentar la guerra defensiva. Muestra una comprensión esencial de la geografía chilena, señalando la línea de costa, la Cordillera de la Costa, el Valle Central y la Cordillera de los Andes. Tan solo se dibujan los ríos Biobío e Imperial. Tiene una desproporción notable en su dimensión longitudinal; el plano se comprime hacia el sur, distorsionando incluso las distancias en leguas consignadas, acabando de modo abrupto en el Golfo de Ancud.

El nombre de Chiloé parece corresponder con un territorio cuyo límite norte se encuentra en el continente y se dibuja mediante línea a trazos. El mapa hace referencia a la ofensiva mapuche dirigida por Pelantaro entre 1598 y 1604, que comienza con la batalla de Curalaba. Esta ofensiva supuso la destrucción del dominio español al sur del Biobío. El plano constituye un registro fiel de estos sucesos. Las ciudades entre Concepción y Castro han sido tachadas, confirmando la nueva situación militar, delimitando seguidamente cinco territorios entre los ríos Biobío y Bueno bajo dominio mapuche. Tres se señalan con su toponimia indígena: Arauco, Catiray y Tucapel. Las ciudades destruidas durante estos sucesos se señalan (consignamos la fecha de su destrucción o abandono entre paréntesis): Valdivia (1599), Angol (1599), La Imperial (1600), Villarrica (1602) y Osorno (1603). No están incluidas otras ciudades eliminadas: Santa Cruz de Coya (1599) y San Andrés de los Infantes (1599), Los Confines (1600) o el fuerte de Santísima Trinidad (1603). Tampoco se muestra la destrucción del fuerte de Arauco (1604), posterior a su viaje, ni el de Cañete. Por otro lado, se marcan poblaciones menores en el plano distribuidas dentro de los territorios bajo dominio mapuche: (Monter[r]ey, Nacimiento, San Gerónimo, Lebo y Paycaví). El plano, aquí, reconstruye el hecho histórico, convirtiéndose en mapa temático que explica esencialmente la dimensión territorial de la derrota militar. Al borrar las plazas y nombrar los territorios mapuche, establece de facto la pérdida del dominio colonial sobre la región. 


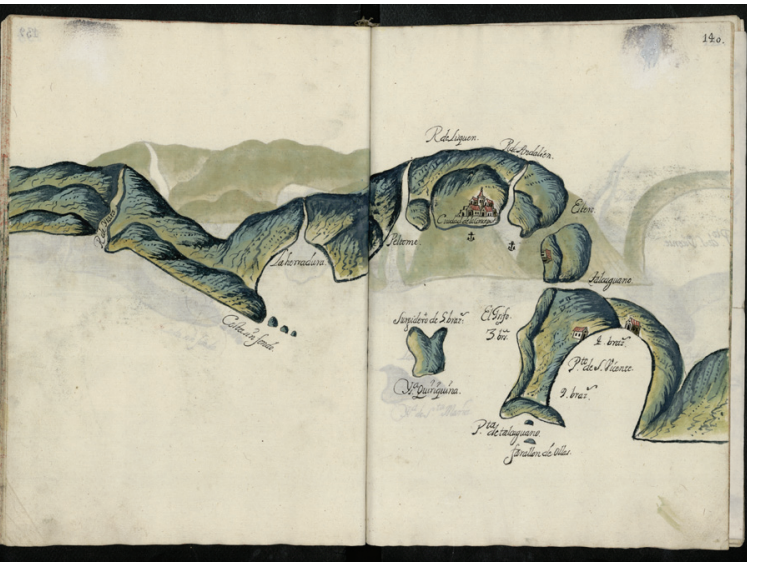

Imagen 5. Mapas de las costas de América en el mar de/ Sur. desde la última población de españoles en ellas, que es la ciudad de Compostela, en adelante. Folio 140 . Fech
(fuente: Biblioteca Nacional de España).

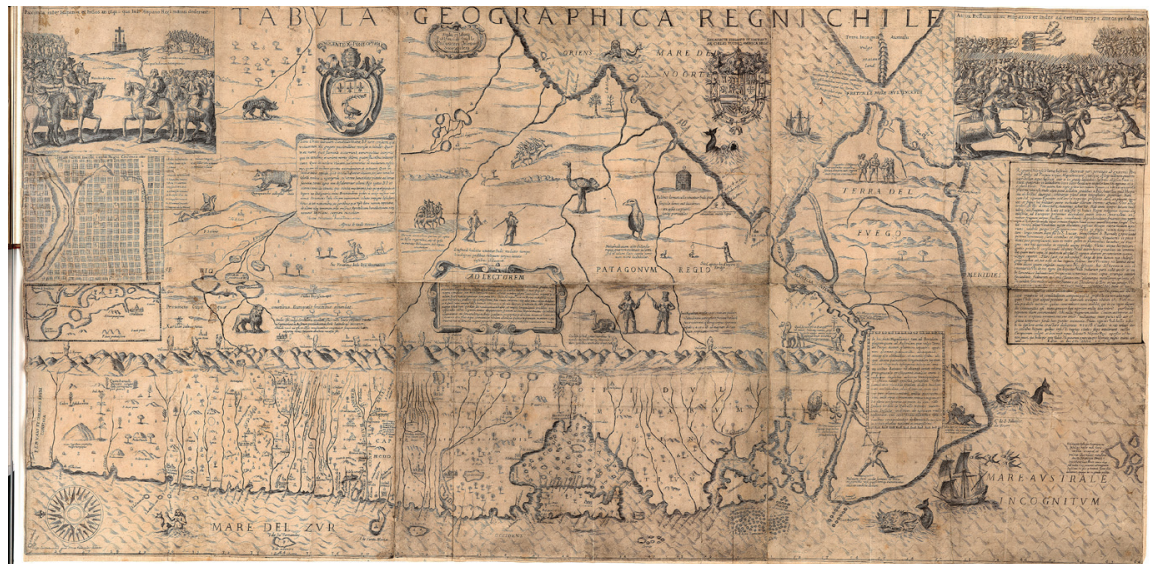

Imagen 6. N4. Tabula geographica Regni Chile/Studio et labore P. Alfonsi de Ovalle, Procuratoris Chilensis Societatis Jesu, 1646 (fuente: John Carter Brown Library).
Este plano ilustra la visión estratégica de la corona española sobre este territorio. Se trata del folio 140 del manuscrito Mapas de las costas de América en el mar del Sur (imagen 5). La copia analizada (quizás el original, entre otras que existen) es un manuscrito del derrotero completo con sus 151 hojas, disponible en la Biblioteca Nacional de España.

Los planos de derroteros de las costas americanas constituían información secreta, propiedad de la corona española. Se trata de mapas instrumentales que ofrecen información valiosa al navegante, permitiéndole situarse con seguridad en costas desconocidas. También aporta la posición de abrigos, fondeaderos, escollos, playas, lugares de aprovisionamiento, poblaciones y, en general, toda información práctica para la navegación. Este derrotero muestra el borde costero occidental de Sudamérica, identificando accidentes geográficos, poblaciones y fondeaderos. Por primera vez se sitúa la ciudad de Concepción (actual Penco) en el contexto territorial inmediato de la bahía, con el cierre de la península de Tumbes (Punta de Talcaguano, Farallón de Ollas), Talcaguano (Talcahuano), puntos de fondeadero, batimetría esencial y esteros (Andalién Lirquén). También se representan las islas
Quiriquina y Rocuant (sin nombrarla) y el Puerto de San Vicente con su profundidad (9 brazas).

Esta y otras cartas posteriores de 1609 , 1619, 1630, 1646-1647, 1650 y 1660 dan fe del interés que las naciones europeas mostraron por el nuevo territorio descubierto y su costa para garantizar la seguridad de sus navegantes y frustrar las aspiraciones de sus competidores. Constituyen planos estratégicos de recursos, seguridad y dominio sobre el territorio.

Un documento fundamental para comprender este período es la carta denominada Tabula geographica Regni Chile, incluida en la Historica relación del Reyno de Chile, de Alonso de Ovalle (1646). La versión más difundida del plano es una reproducción reducida de $37 \times 17,5 \mathrm{~cm}$ del grabado original de $47 \times 36 \mathrm{~cm}$. Este es, a su vez, una versión reducida y simplificada de un plano de mayor tamaño, formado por seis hojas, de dimensiones mayores (116,3 $\times 57,4 \mathrm{~cm}$ ). Existen tres ejemplares de este plano: dos en la Bibliothèque nationale de France y el aquí presente (imagen 6). El plano grande reconoce aspectos importantes que no aparecen en las versiones reducidas. En él se traza el Cono Sur americano desde el río Salado hasta Tierra del Fuego entre 25 y 54 grados de latitud. El conocimiento de la geografía de la región es notable, aunque muestra una diferencia clara entre la fiabilidad del conocimiento al norte y al sur del río Biobío, sin reconocer lagos en la precordillera y localizando erróneamente algunos afluentes. Más allá de ser la descripción física más completa hasta entonces del territorio chileno, el interés de este mapa radica en confirmar la posición del río Biobío como frontera entre la corona española y el pueblo Mapuche tras el Parlamento de Quilín de 1641. El plano marca la frontera del río Biobío mediante el uso de sendos textos situados en la ribera norte y sur del río donde se lee Præsidium hispanorum (defensa de los españoles) y Confinia belli (fronteras de guerra) respectivamente. El texto refleja la consolidación del espacio fronterizo y el confinamiento de la empresa colonial. Esta delimitación se refuerza situando cada fuerte de la línea de frontera. Cabe destacar que, a pesar de haber sido destruidas y despobladas, el plano identifica las ciudades al sur del Biobío. También señala los yacimientos de oro en territorio mapuche. Implícitamente, da cuenta de una situación que espera que se revierta y donde se siguen estudiando los recursos del territorio para su explotación futura. 
CONCLUSIONES. Cartografiar el territorio consiste en representar una información selecta de modo convencional. Tanto los elementos representados como los códigos de representación permiten una doble lectura: por un lado, 'declaran' lecturas explícitas del territorio que pretenden ser neutras. Por otro lado, 'revelan' entornos, intereses y sistemas de valores construidos desde el poder. Estos planos identifican claves territoriales para orientar la exploración y explotación de la región. El conocimiento de la geografía es creciente, si bien hay un quiebre profundo a partir de 1605 . Hasta 1646 no vuelve a reconocerse este territorio (tarea que no se completará hasta finales del siglo XIX). La consecuencia directa es la paralización de la acción militar. A partir de entonces, los misioneros y clérigos toman protagonismo en el conocimiento territorial araucano. Esta circunstancia constituye un factor fundamental de preservación del paisaje mapuche hasta la ocupación de la época republicana. Prueba de este período de impasse es la cartografía del período, donde se aprecian importantes lagunas, errores e indeterminaciones que atestiguan la poca información con la que se cuenta. Sin embargo, no se renuncia a la lógica de conquista y se siguen representando los elementos que la soportaron en su inicio, incluso aunque hayan desaparecido. Este desconocimiento prolongado del territorio conduce a una idealización mítica del paisaje. Testimonios como los de Ocaña y Ovalle contribuyen a la consolidación de la idea de un territorio indómito, idea que perdurará durante tres siglos.

\section{REFERENCIAS}

Alfaro, L., 2014. Los reche-mapuche a través de su sistema de asentamiento (s. XV-XVII). Tesis para optar al grado de doctor. Universidad de Chile. Disponible en: http://www.academia.edu/26495579/Los_rechemapuche_a_trav\%C3\%A9s_de_su_sistema_de_asentamiento_s._XV-XVII_

Aliste, E., 2011. "Territorio y huellas territoriales: una memoria del espacio vivido en el Gran Concepción, Chile." Desenvo/vimento e Meio Ambiente, 23, 25-38. Disponible en: htto://repositorio.uchile.cl/handle/2250/117884 Álvarez, L., 2000. "Cartografia y geodesia: las innovaciones de la Oficina de Mensura de Tierras de Chile a principios del siglo XX (1907-1914)." Scripta Nova. Revista Electrónica de Geografía y Ciencias Sociales, 69 (12) Disponible en: http://www.ub edu/geocrit/sn-69-12.htm

Antivil, W. 2018. Dibuiando la Araucanía: la construcción, la forma y el dominio de un territorio. Tesis para optar al grado de doctor. Universidad Politécnica de Cataluña. Disponible en: https://upcommons.upc.edu/ handle/2117/115032? show=full

Archivo General de Indias, 1610. Mapa del Reino de Chile. Mapa, manuscrito. Archivo General de Indias. Biblioteca Nacional de España, s/f., Mapas de las costas de América en el mar del Sur, desde la uultima población de españoles en ellas, que es la ciudad de Compostela, en adelante. Manuscrito. Biblioteca Nacional de España. Disponible en: http://bdh-rd.bne.es/viewer.vm?id=0000117007\&page=

Biblioteca Nacional de España, s/f. Mapa del territorio comprendido entre el rio Itata y el Golfo de Arauco s. XVII. Mapa, manuscrito. En Mapas de las costas de América en el mar del Sur, desde la última población de españoles en ellas, que es la ciudad de Compostela, en adelante. Manuscrito. Folio 140.

Bousquet-Bressolier, C., 1995. "L'ㄷil du cartographe et la représentation géographique du Moyen Age à nos jours." (Coll. «Mémoires de la section de géographie physique et humaine »). Dix-huitième Siècle, 30, 635. Disponible en: http://www.persee.fr/doc/dhs_00706760_1998_num_30_1_2266_t1_0635_0000_2 Cárcel, C., 2016. "La cartografía histórica como método de estudio morfológico del paisaje tradicional de Campanar" EGA Revista de expresión gráfica arquitectónica, 21 (27): 254-261.

Disponible en: https://polipapers.upves/index.php/EGA/article/view/4745

Cieza de León, P., 1554. Brevis exactaque totius Novi Orbis eiusque insularum descriptio recens a Joan. Bellero edita. Mapa, xilografía15 x $10 \mathrm{~cm}$. Amberes: The John Carter Brown Library Map Collection (EEUU).

Cisternas, M. y Torrejón, F., 2002. "Cambios de uso del suelo, actividades agropecuarias e intervención

ambiental temprana en una localidad fronteriza de la Araucania (s. XVI-XIX)." Revista de Geografía Norte Grande. 29, 83-94. Disponible en: http://revistanortegrande.cl/archivos/29/06 29 2002.pdf

Cosgrove, D. y Daniels, S. (Eds.)., 1988. The iconography of landscape: essays on the symbolic representation design and use of past environments. Cambridge/Nueva York: Cambridge University Press.

Dillehay, T. y Saavedra, J., 2010. Los valles de Purén-Lumaco y Liucura, Chile: arqueología e historia cultural. Vanderbilt University Disponible en: https://discoverarchivevanderbiltedu/bitstream/handle/1803/5795/ VUPA52. pdf? sequence=1

Edney, M., 2007. Recent trends in the history of cartography: A selective, annotated bibliography to the English-language literature. Series B. No 6. MAGERT - ALA Map and Geography Round Table. Disponible en: http://oaktrustlibrarytamuedu/handle/1969.1/129196

Flores, J. y Azócar, A., 2017. "Mapas para el Estado. La representación de la Araucanía: 1836-1916." Scripta Nova. Revista Electrónica de Geografia y Ciencias Sociales, 21 (562): 1-25. Disponible en: http://www.raco.cat/ index.php/ScriptaNova/article/view/321421

González, J. y Bernedo, P.. 2013. "Cartografía de la transformación de un territorio: La Araucanía 1852-1887. Revista de Geografia Norte Grande, 54, 179-198. Disponible en: https://scielo.conicyt.cl/scielo.php?script=sci_ar ttext\&pid=S0718-34022013000100010

Guarda, G., 1990. Flandes indiano: las fortificaciones del Reino de Chile, 1547-1826. Santiago de Chile: Ediciones Universidad Católica de Chile.

Guarda, G., 1978. Historia urbana del Reino de Chile. Santiago de Chile: Andres Bello.
Harley, J., Laxton, P. y Andrews, J., 2005. La nueva natura/eza de los mapas: Ensayos sobre la historia de la cartografía. Ciudad de México: Fondo de Cultura Económica.

Kagan, R., 2000. "Cartografia y comunidad en el mundo hispánico." Pedralbes: revista d'história moderna, 20 11-36. Disponible en: https://dialnet.unirioja.es/servlet/articulo?codigo=1112904

Kaplan, R., 2013. La venganza de la geografía. Cómo los mapas condicionan el destino de una nación. Barcelona: RBA.

Lefebvre, H., 1974. "La producción del espacio." Papers: revista de sociología, 3, 219-229. Disponible en: www raco.cat/indexphp/Papers/article/download/52729/60536

Lois, C., 2000. "La elocuencia de los mapas: un enfoque semiológico para el análisis de cartografías" Documents d'Analisi Geográfica, 36, 93-109. Disponible en: http://www.raco.cat/index.php/DocumentsAnalisi/ article/view/31715

López de Velasco J y Fernández, C, 1894 Geografía y descripción universal de las Indias. recopilada por el cosmógrafo-cronista Juan López de Velasco desde el año de 1571 al de 1574: publicada por primera vez en el Boletin de la Sociedad Geográfica de Madrid, con adiciones é ilustraciones, por don Justo Zaragoza. Establ. tip. de Fortanet. Disponible en: http://www.cervantesvirtual.com/obra/geografa-y-descripcin-universal-de-lasindias-recopilada-por-el-cosmgrafocronista-iuan-|pez-de-velasco-desde-el-ao-de-1571-al-de-1574-publicada-por-d-justo-zaragoza-0/

López de Velasco, J., 1601. Descripcion de Prouincia de Chile. Mapa, manuscrito. 14,5 X 28,9 cm. The John Carter Brown Library Map Collection (EEUU).

Maderuelo, J., 2005. El paisaje: génesis de un concepto. Madrid: Abada Editores

Núñez, A. 2012. "El país de las cuencas: fronteras en movimiento e imaginarios territoriales en la construcción de la nación. Chile siglos XVIII-XIX." Scripta Nova. Revista Electrónica de Geografía y Ciencias Sociales, 16 (418). Disponible en: http://www.ub.edu/geocrit/sn/sn-418/sn-418-15.htm

Ocaña, D. de., s/f.. Relación del viaje de Fray Diego de Ocaña por el Nuevo Mundo (1599-1605). Manuscrito. Disponible en: http://digibuo.uniovi.es/dspace/handle/10651/27859

Ocaña, D. 1605. Mapa del territorio comprendido entre los ríos Biobio y Cautín. Mapa, manuscrito. En Relación del viaje de Fray Diego de Ocaña por el Nuevo Mundo (1599-1605). 1605. Manuscrito. Folio 85. Biblioteca de la Universidad de Oviedo, España.

Ovalle, A. de., 1646. Histórica relación del Reyno de Chile Y de las misiones y ministerios que exercita en la Compañía de Jesús A Nuestro Señor Jesu Christo Dios Hombre Y a la Santíssima Virgen y Madre María Señora del Cielo, y de la Tierra y a los Santos Joseph, Joaquin, Ana sus Padres y Abuelos Alonso de Ovalle de la Compañía de Jesús, natural de Santiago de Chile, y su Procurador en Roma. Francisco Caballo. Ovalle, A. de., 1646. Tabula geographica Regni Chile. Mapa. 57,4 X 116,3 cm. Grabado. Roma. Francisco Cauallo (Ed.), John Carter Brown Library.

Peña, B., 2016. Fray Diego de Ocaña: olvido, mentira y memoria. Alicante: Universidad de Alicante. Disponible en: http://rua.ua.es/dspace/handle/10045/53475

Sagredo, R., 2013. "De la hidrografía imperial a la hidrografía nacional. Reconocimientos del Pacífico sur. Siglos XVIII y XIX." Anuario de Estudios Americanos, 70, 509-556. Disponible en: http://historia.uc.cl/images/stories/ publicaciones/rsagredo/de\%20la\%20hidrografa\%20imperial\%20a\%20la.pdf

San-Antonio, C. 2006. "Metodología para el análisis gráfico de la cartografía histórica." En XVIII Congreso Internacional de Ingeniería Gráfica: diseño e innovación: Actas del congreso. Sitges: INGEGRAF Universidad Politècnica de Catalunya, 81-95

Solari, M., Cueto, C., Hernández, F., Rojas, J. y Camus, P., 2011. "Procesos territoriales y bosques en la cuenca del río Valdivia (siglos XVI-XIX)." Revista de Geografía Norte Grande, 49: 45-62. Disponible en: https://scielo. conicyt.cl/scielo.php?script=sci_arttext\&pid=S0718-34022011000200004

Wroth, L. C. 1959. Alonso de Ovalle's large map of Chile, 1646. Imago Mundi, 14, 90-95. Disponible en: https:/ wwwistororg/stable/1150215?seq=1\#page scan tab contents 